(c) American Dairy Science Association, 2006.

\title{
Preparation of Liposomes from Milk Fat Globule Membrane Phospholipids Using a Microfluidizer
}

\author{
A. K. Thompson and H. Singh ${ }^{1}$ \\ Riddet Centre, Massey University, Private Bag 11 222, Palmerston North, New Zealand
}

\begin{abstract}
The isolation of milk fat globule membrane (MFGM) material from buttermilk on a commercial scale has provided a new ingredient rich in phospholipids and sphingolipids. An MFGM-derived phospholipid fraction was used to produce liposomes via a high-pressure homogenizer (Microfluidizer). This technique does not require the use of solvents or detergents, and is suitable for use in the food industry. The liposome dispersion had an average hydrodynamic diameter of $95 \mathrm{~nm}$, with a broad particle-size distribution. Increasing the number of passes through the Microfluidizer, increasing the pressure, or reducing the phospholipid concentration all resulted in a smaller average liposome diameter. Changing these variables did not have a significant effect on the polydispersity of the dispersion. Electron microscopy showed that the dispersions formed had a range of structures, including unilamellar, multilamellar, and multivesicular liposomes. The composition of the MFGM phospholipid material is different from that of the phospholipids usually used for liposome production in the pharmaceutical and cosmetic industries. The MFGM-derived fraction comprises approximately $25 \%$ sphingomyelin, and the fatty acids are primarily saturated and monounsaturated. These differences are likely to affect the properties of the liposomes produced from the phospholipid material, and it may be possible to exploit the unique composition of the MFGM phospholipid fraction in the delivery of bioactive ingredients in functional foods.
\end{abstract}

Key words: milk fat globule membrane, liposome, encapsulation, Microfluidizer

\section{INTRODUCTION}

Whole milk contains approximately $0.035 \%$ phospholipid, of which approximately $35 \%$ is in the milk serum, and the remaining $65 \%$ is in the milk fat globule membrane (MFGM). The MFGM phospholipids are primar-

Received August 30, 2005.

Accepted October 4, 2005.

${ }^{1}$ Corresponding author: h.singh@massey.ac.nz ily phosphatidyl choline (PC), phosphatidyl ethanolamine (PE), and sphingomyelin (SM), with small amounts of phosphatidyl serine and phosphatidyl inositol (PI).

Phospholipids have been shown to have a number of health benefits, including liver protection (Koopman et al., 1985) and memory improvement (Crook et al., 1991, 1992). Sphingolipids are required for cellular signaling, and have been shown to be involved in the control of cell proliferation, apoptosis, inflammation, and cancer (Huwiler et al., 2000). Sphingomyelin also inhibits intestinal absorption of cholesterol and fat in rats, with milk SM being more effective than egg SM (Peel, 1999). Sphingolipids are traditionally extracted from bovine brain, and are not only very expensive but also unsuitable for use in foods for vegetarians. Issues relating to bovine spongiform encephalopathy and CreutzfeldtJakob disease make it undesirable to use material extracted from bovine brain in food systems. The relatively high concentrations of sphingolipids in dairy phospholipid could avoid many of the negative issues surrounding many of the other sources of phospholipids and sphingolipids.

The identification of these biological functions of phospholipids, in particular sphingolipids, has led to increasing interest in techniques for isolating phospholipid fractions from waste dairy streams, such as buttermilk. These techniques range from traditional methods using solvent extraction to emerging technologies such as microfiltration and supercritical fluid extraction (Astaire et al., 2003; Corredig et al., 2003). Currently, the Fonterra Cooperative Group Ltd. (New Zealand) is the only company known to extract and purify MFGM phospholipid fractions from buttermilk commercially. In addition to their biological functions, MFGM phospholipids have been to shown to have good emulsification properties (Corredig and Dalgleish, 1998; Roesch et al., 2004), and have been used for the production of emulsions for drug delivery (Sato et al., 1994; Yuasa et al., 1994).

In the pharmaceutical and cosmetic industries, highly purified phospholipids extracted from soy oil or egg yolk are used to produce liposomes. Liposomes are spherical structures consisting of one or more phospho- 
lipid bilayers enclosing an aqueous core (Zeisig and Cämmerer, 2001). They may be used for the entrapment and controlled release of drugs or nutraceuticals, as model membranes or cells, and for specialist applications such as gene delivery (Lasic, 1998).

There are many potential applications for liposomes in the food industry, ranging from the protection of sensitive ingredients to increasing the efficacy of food additives. However, the high cost of the purified soy and egg phospholipids, combined with problems in finding a production method suitable for use in the food industry, has limited the use of liposomes in foods. To our knowledge, MFGM-derived phospholipids have never been used in making liposomes. The high levels of sphingolipids in MFGM phospholipids may provide nutritional benefit for the consumer, as well as improved liposome functionality.

There are several methods that may be used to produce liposomes, and a number of excellent reviews have been published that provide preparation details of the more common production techniques (Watwe and Bellare, 1995; Betageri and Kulkarni, 1999; Frezard, 1999). The standard preparation procedure is via the rotary evaporation of a chloroform solution of phospholipid, cholesterol, and other hydrophobic compounds to produce a thin phospholipid film. Addition of water and hydrophilic compounds causes bilayer sheets of the lipid to separate from the bulk and form liposomes (Picon et al., 1994). Jackson and Lee (1991) stated that the largescale production of liposomes was limited by poor encapsulation efficiencies, the lack of a continuous production process, and the use of organic solvents. They concluded that the solution to this problem might be the use of a microfluidization technique. The Microfluidizer is a high-pressure homogenizer that can rapidly produce a large volume of liposomes in a continuous and reproducible manner (Chen et al., 2001), without use of sonication, detergents, solvents, or alcohols. The liposome population produced appears to be relatively stable, without rapid aggregation or fusion (Kim and Baianu, 1991). In microfluidization, the phospholipid and the material to be entrapped are dispersed in a liquid phase. This may be water, an aqueous buffer solution, or a solvent, depending on the solubilities of the components. The solution is pressurized in continuous flow, and split into 2 streams that are then forced together at high velocity $(>500 \mathrm{~m} / \mathrm{s})$. The resulting release of kinetic energy provides the required activation energy to break up the large phospholipid bilayer sheets into smaller fragments (Kim and Baianu, 1991). To minimize surface energy, the ends wrap around, forming bilayer vesicles known as liposomes.

The objective of the research presented in this paper was to study the formation of liposomes using a mi- crofluidization technique. A phospholipid-rich fraction isolated on an industrial scale from MFGM was used to prepare liposomes.

\section{MATERIALS AND METHODS}

A phospholipid-rich fraction derived from MFGM (Phospholac 600) was provided by the Fonterra Cooperative Group Ltd. (New Zealand). It contained approximately $83 \%$ lipid, $6.2 \%$ lactose, $11.5 \%$ ash, and $2.6 \%$ moisture. All chemicals and other materials used were of analytical grade and were obtained from Sigma-Aldrich (St. Louis, MO).

\section{Fatty Acid Profile of Phospholipid Fraction}

The lipid was extracted from the phospholipid fraction using chloroform and methanol in a ratio of 1:2 (by volume). Fatty acids were methylated by acid-catalyzed transesterification at $80^{\circ} \mathrm{C}$ for $12 \mathrm{~h}$ in a sealed tube. The fatty acid methyl esters were separated using a BPX-70 capillary column, $100 \mathrm{~m} \times 0.22 \mathrm{~mm}$ i.d., 0.25 $\mu \mathrm{m}$ film (SGE, Melbourne, Australia). The gas chromatographic system consisted of a model 6890 gas chromatograph equipped with an autosampler (HP7673) and Chem Station integration (all Hewlett Packard, Avondale, PA). The column oven was held at an initial temperature of $165^{\circ} \mathrm{C}$ for $52 \mathrm{~min}$, and then increased at a rate of $5^{\circ} \mathrm{C} / \mathrm{min}$ to a final temperature of $210^{\circ} \mathrm{C}$ for $59 \mathrm{~min}$ (total run time: $120 \mathrm{~min}$ ). Both the injector port and the flame-ionization detector port were at $250^{\circ} \mathrm{C}$. The carrier gas flow (helium) was maintained at 1.0 $\mathrm{mL} / \mathrm{min}$ (linear gas velocity: $20 \mathrm{~cm} / \mathrm{s}$ ) throughout the temperature program with an inlet split ratio of 30:1. Fatty acid peaks were identified by retention time matching with authentic standards, including a composite standard made from commercially available methyl esters (NuChek Prep, Elysian, MN; Sigma, St. Louis, MO).

\section{Analysis of Phospholipid Head Group}

${ }^{31}$ Phosphorus-nuclear magnetic resonance $\left({ }^{31} \mathrm{P}\right.$ NMR) analysis was conducted on the MFGM phospholipid material in its original powder state. The analysis was performed by Spectral Service (Köln, Germany) using a Bruker AC-P $300-\mathrm{MHz}$ NMR spectrometer (Diehl, 2001, 2002).

\section{Preparation of Liposome Dispersions}

A $10 \%$ lipid dispersion was made in imidazole buffer (20 mM imidazole, $50 \mathrm{~m} M$ sodium chloride, and $0.02 \%$ sodium azide in Milli-Q water, adjusted to $\mathrm{pH} 7$, with 
$1 M \mathrm{HCl}$ ) and thoroughly mixed using an Ultra-Turrax blender (JKA, Staufen, Germany). The phospholipid dispersion was then processed using an M-110Y Microfluidizer (Microfluidics International Corp., Newton, MA) with a 75- $\mu \mathrm{m}$ F12Y-type interaction chamber (Talsma et al., 1989; Larivière et al., 1991).

\section{Determination of Size and Size Distribution of Liposomes}

The average hydrodynamic diameter of the liposome dispersions was measured on a Zetasizer 4 (Malvern Instruments Ltd., Worcestershire, UK) using photon correlation spectrometry. Samples of the liposome dispersions were diluted in imidazole buffer to the required turbidity $(<250 \mathrm{kilocounts} / \mathrm{s})$. Preliminary experiments had found no effect of concentration on the measured diameter at turbidities of up to $500 \mathrm{kcps}$. Each sample was analyzed 3 times at $25^{\circ} \mathrm{C}$ with a sampling time of $99 \mathrm{~s}$ and a scattering angle of $90^{\circ}$. A medium viscosity of $1.054 \mathrm{cP}$ and a refractive index of 1.34 were used for the aqueous phase, with a typical liposome refractive index of 1.45 (Blessing et al., 1998; Ardhammer et al., 2002).

Asymmetrical flow field-flow fractionation was used to provide more detailed information regarding the size distribution of the liposome dispersions. This was performed using a Postnova Avalanche AF4 AFFF (Postnova, Munich, Germany) equipped with a Postnova refractive index detector (PN 3140) and a PD Expert multiangle dynamic and static light-scattering system (Precision Detectors, Bellingham, MA). A channel spacer of $0.25 \mathrm{~mm}$ and a field programming method using power field decay were used. The field was initially held constant at $70 \%$ for $4.8 \mathrm{~min}$, and then decayed at the rate of $-\mathrm{p} \times 4.8 \mathrm{~min}$, where $P=2$ (to obtain a constant fractionation power). This method was designed using the software provided with the system to give the optimum separation (in terms of maximum resolution) in 60 min for particles between 7.5 and 750 $\mathrm{nm}$ in diameter. The crossflow and the expected particle size of eluted material as a function of time are shown in Figure 1. The channel outlet flow was held constant at $0.3 \mathrm{~mL} / \mathrm{min}$ and the total run time was $60 \mathrm{~min}$.

\section{Effect of Processing Variables on Average Hydrodynamic Diameter of Liposome Dispersions}

An experiment was conducted to determine the effects of the number of passes through the interaction chamber and the Microfluidizer operating pressure or the concentration of the phospholipid dispersion on the size of the liposomes produced. Phospholipid dispersions of 1,5 , and $10 \%$ phospholipid (wt/wt) were cycled

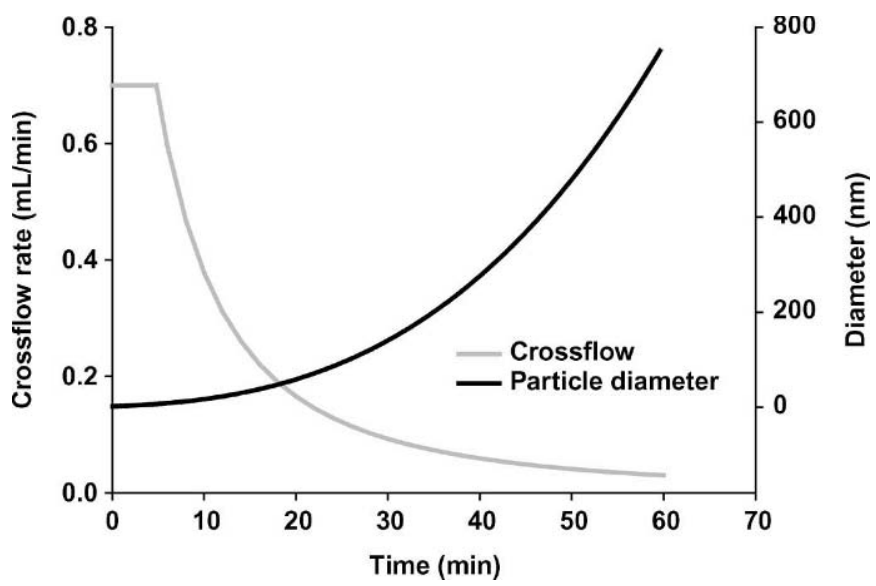

Figure 1. Change in crossflow rate and eluted particle diameter over time resulting from programming conditions used for asymmetrical flow field-flow fractionation.

through the Microfluidizer up to 10 times at pressures of 73,90 , and $103 \mathrm{MPa}(10,000$ to $17,000 \mathrm{psi}$ ), and liposome size and polydispersity were measured using the Zetasizer 4 as outlined above. At least 3 replicates were made for each sample.

\section{Determination of $\zeta$-Potential}

Liposome dispersions were prepared using $0.1 \mathrm{M}$ $\mathrm{NaCl}$, and the $\mathrm{pH}$ was adjusted using $1 M \mathrm{HCl}$ and 1 $M \mathrm{NaOH}$. The samples were diluted as required using $0.1 M \mathrm{NaCl}$ solution that had been adjusted to the specific $\mathrm{pH}$. The $\zeta$-potential was measured using the Zetasizer 4 (Malvern Instruments Ltd.) with an AZ104 cell. Five measurements of $25 \mathrm{~s}$ duration at $100 \mathrm{mV}$ were used to measure the $\zeta$-potential at the stationary layer $14.63 \%$ of the capillary diameter in from the wall.

\section{Negative-Staining Transmission Electron Microscopy}

The liposome dispersions were diluted approximately 1:10 with distilled water. One drop of the diluted sample was combined with a drop of $2 \%$ ammonium molybdate and left for $3 \mathrm{~min}$. The solution was then placed on a copper mesh for $5 \mathrm{~min}$ before the excess liquid was drawn off with filter paper. The mesh was examined using a Philips 201C transmission electron microscope (Eindhoven, The Netherlands).

\section{Thin-Section Transmission Electron Microscopy}

The liposome dispersion was mixed with low-temperature gelling agarose. The agarose-embedded samples were cut into $\sim 1 \mathrm{~mm}^{3}$ cubes, placed in a bijoux bottle 
containing $3 \%$ glutaraldehyde in $0.2 \mathrm{M}$ sodium cacodylate buffer, and kept at $5^{\circ} \mathrm{C}$ for $24 \mathrm{~h}$. The glutaraldehyde was removed by rinsing twice with $0.2 \mathrm{M}$ sodium cacodylate buffer for $2 \mathrm{~h}$, and then the samples were left in $1 \%$ osmium tetroxide overnight at room temperature. The samples were washed twice with distilled water, placed in $1 \%$ uranyl acetate for $30 \mathrm{~min}$, and then washed twice more with distilled water.

The embedded samples were dehydrated at $5^{\circ} \mathrm{C}$ using $25 \%$ acetone for $15 \mathrm{~min}$, and then 50, 70, and $90 \%$ acetone for $30 \mathrm{~min}$ each, followed by $100 \%$ acetone. The acetone was replaced with Procure 812 embedding resin, and the samples were put on rollers for $24 \mathrm{~h}$. A cube of each sample was placed into an embedding capsule, which was then cured at $60^{\circ} \mathrm{C}$ for $48 \mathrm{~h}$.

The samples were then sectioned to a thickness of 90 $\mathrm{nm}$ using a Reichert Ultracut microtome. These sections were mounted on 3-mm copper grids and stained with lead citrate before examination in a Philips 201C transmission electron microscope at an accelerating voltage of $60 \mathrm{kV}$.

\section{Cryo-Field Emission Scanning Electron Microscopy}

Samples of liposome dispersions were plunge-frozen using liquid propane, before being frozen with slushy liquid nitrogen at $-140^{\circ} \mathrm{C}$. They were then fractured with a knife on an Alto 2500 cryo stage (Gatan, Abingdon, UK), and freeze-etched by raising the temperature from -140 to $-90^{\circ} \mathrm{C}$ before returning to $-140^{\circ} \mathrm{C}$. The exposed surface was then coated with $\mathrm{Au}$ and $\mathrm{Pd}$ for $120 \mathrm{~s}$, before being examined under a JSM-6700F field emission scanning electron microscope (JEOL, Tokyo, Japan). An accelerating voltage of $10 \mathrm{kV}$ was used.

\section{Determination of Phospholipid Oxidation}

The peroxide value and the level of conjugated dienes were measured before and after microfluidization to provide an indication of the effect of the processing on phospholipid oxidation. The peroxide value was obtained using a technique based on ISO 3960:2001. Briefly, $1 \mathrm{~mL}$ of the liposome dispersion was dissolved in $6 \mathrm{~mL}$ of acetic acid:chloroform (3:2), and $0.5 \mathrm{~mL}$ of saturated potassium iodide solution was added. The sample was mixed for $1 \mathrm{~min}$, with further addition of $6 \mathrm{~mL}$ of distilled water. The solution was titrated against $0.1 \mathrm{M}$ sodium thiosulfate solution until the yellow color had almost disappeared. Then, $0.5 \mathrm{~mL}$ of a $1.0 \%$ starch solution was added, and titration was continued until the blue color disappeared. The peroxide value was calculated as milliequivalents of peroxide per $1,000 \mathrm{~g}$ of sample.
Table 1. Comparison between the proportions of phospholipid classes in the milk fat globule membrane (MFGM)-derived phospholipid fraction and published values for MFGM phospholipids

\begin{tabular}{lll}
\hline & $\begin{array}{l}\text { MFGM-derived } \\
\text { phospholipid } \\
\text { fraction } \\
(\%)\end{array}$ & $\begin{array}{l}\text { Published } \\
\text { values for } \\
\text { MFGM } \\
\text { phospholipids }\end{array}$ \\
Lipid & 72 & $(\%)$ \\
\hline Phospholipid & 32.8 & - \\
Phosphatidyl choline & 28.1 & 36 \\
Phosphatidyl ethanolamine & 31.7 & 27 \\
Sphingomyelin & 3.5 & 22 \\
Phosphatidyl serine & 3.6 & 4 \\
Phosphatidyl inositol & 0.3 & 11 \\
Other phospholipids & & - \\
\hline
\end{tabular}

${ }^{1}$ Keenan and Mather, 2002.

The levels of conjugated dienes and trienes formed during oxidation were determined by a method based on IUPAC method no. 2.505 (IUPAC, 1987) and Lethuaut et al. (2002). A 25- $\mu \mathrm{L}$ aliquot of the liposome dispersion was dissolved in $10 \mathrm{~mL}$ of isopropanol, mixed for $4 \mathrm{~s}$, and centrifuged for $5 \mathrm{~min}$ at 2,500 $\times g($ CentraMP4R centrifuge, International Equipment Company, Needham Heights, MA). The absorbance was read against a blank containing $10 \mathrm{~mL}$ of isopropanol and $25 \mu \mathrm{L}$ of Milli-Q water at $232 \mathrm{~nm}$ (linoleic hydroperoxides and conjugated dienes) and $268 \mathrm{~nm}$ (conjugated trienes and secondary products).

\section{RESULTS AND DISCUSSION}

\section{Composition of MFGM Phospholipid Material}

The phospholipid head group analysis by ${ }^{31} \mathrm{P}$-nuclear magnetic resonance analysis showed that the dairyderived phospholipid fraction used in this work comprised $\sim 72 \%$ phospholipid. This phospholipid material contained between 28 and $33 \%$ each of PC, PE, and SM, and $\sim 3.5 \%$ each of PI and phosphatidyl serine (Table 1). This is in general agreement with previous reports on the phospholipid composition of MFGM (Boyd et al., 1999; Keenan and Mather, 2002; Astaire et al., 2003). This composition is considerably different from that of phospholipids derived from other sources. For example, the phospolipid products manufactured by Avanti Polar Lipids (Alabaster, AL) derived from egg yolk are composed almost entirely of PC, with small amounts of PE and PI, and a very small amount of SM. Soy phospholipid material may contain PC, PE, and PI, but does not contain any SM.

These differences in phospholipid composition would be expected to affect a number of liposome characteristics, including surface charge, resistance to destabilizing influences (i.e., high ionic concentration), and membrane permeability. The dairy phospholipid fraction 
Table 2. Comparison between the polar lipid composition of the milk fat globule membrane (MFGM)-derived phospholipid fraction and published values for MFGM

\begin{tabular}{lcc}
\hline & $\begin{array}{l}\text { MFGM-derived } \\
\text { phospholipid } \\
\text { fraction } \\
(\%)\end{array}$ & $\begin{array}{l}\text { Published } \\
\text { values for } \\
\text { MFGM }\end{array}$ \\
Fatty acid & 3.1 & $(\%)$ \\
\hline C14:0 & 16.2 & $1.9-2.2$ \\
C16:0 & 1.4 & $16.5-19.4$ \\
C16:1 & 10.0 & $1.2-1.6$ \\
C18:0 & 30.3 & $16.3-17.9$ \\
C18:1 & 4.8 & $27.7-30.7$ \\
C18:2n-6 & 1.8 & $6.1-6.9$ \\
C18:3n-3 & 0.5 & $0.4-0.6$ \\
C20:2n-6 & 0.7 & $0.5-1.5$ \\
C20:3n-6 & 0.7 & $1.0-1.3$ \\
C20:4n-6 & 0.6 & $0.9-2.2$ \\
C20:5n-3 & 4.2 & $0.2-0.6$ \\
C22:0 & 1.0 & $3.5-4.8$ \\
C22:5n-3 & 6.5 & $0.5-0.6$ \\
C22:5n-6 & 4.0 & $0.3-0.5$ \\
C24:0 & 2.6 & $3.1-3.9$ \\
Other $(<0.3 \%)$ & 11.5 & - \\
Unknown & & - \\
\hline
\end{tabular}

${ }^{1}$ Fauquant et al., 2005.

also contained relatively high amounts of SM, and it has been shown that phospholipid bilayers containing SM appear to be more stable and have a lower permeability to hydrophilic molecules than PC bilayers (New, 1990b).

The fatty acid profile of the phospholipid fraction is shown in Table 2. As expected, this fraction contained relatively high proportions of saturated and monounsaturated fatty acids, with palmitic acid (16:0), stearic acid (18:0), and oleic acid (18:1) being the most common. These values are in general agreement with those reported by Fauquant et al. (2005) for the fatty acid profile of MFGM; however, there was a significantly higher proportion of $\mathrm{C} 22: 5$ fatty acid chains than reported in the literature. This may be a consequence of the selective removal of buttermilk components during fractionation. In contrast, the phospholipids of soy or egg origin tend to have a higher proportion of mono- and polyunsaturated fatty acids, and are predominantly composed of palmitic acid (16:0), oleic acid (18:1), and linoleic acid (18:2) (Weiner, 1995; Pheko et al., 1998).

The types of fatty acids present in a liposome membrane affect the packing of the lipid bilayer (New, 1990a). Consequently, liposomes containing a higher proportion of saturated fatty acids may have a higher phase transition temperature, lower membrane permeability, and reduced susceptibility to oxidation compared with membranes composed of more highly unsaturated fatty acids.

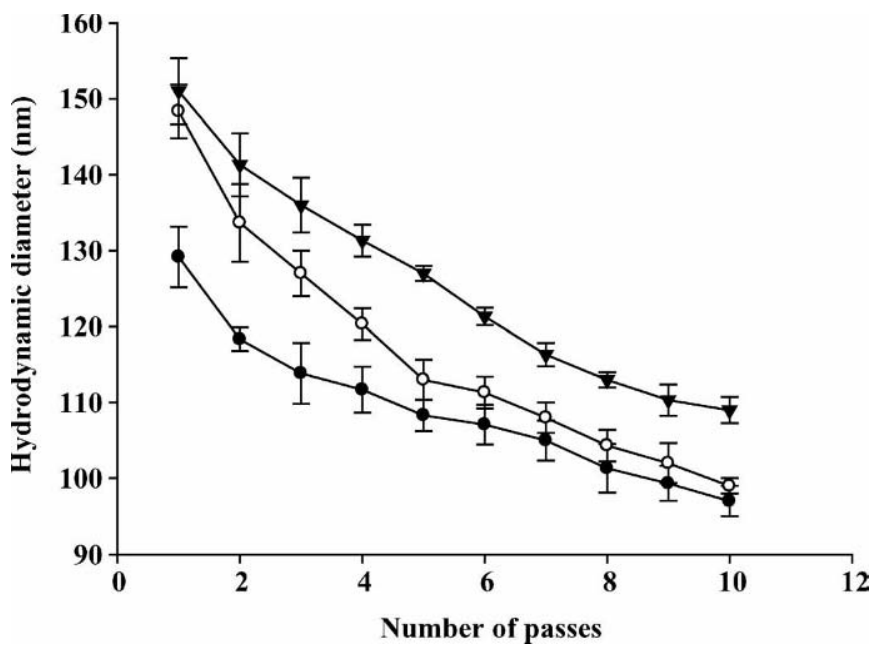

Figure 2. Effect of Microfluidizer pressure on average hydrodynamic diameter for a $10 \%$ phospholipid dispersion. The phospholipid dispersions were treated at $103 \mathrm{MPa}(\boldsymbol{\nabla}), 90 \mathrm{MPa}(\bigcirc)$, and $73 \mathrm{MPa}$

(๑). Each data point is the average of 3 or more replicates.

\section{Particle Size and Size Distribution}

The first pass through the Microfluidizer had the largest effect on liposome size, resulting in a decrease in average hydrodynamic diameter from over $500 \mathrm{~nm}$ to between 100 and $150 \mathrm{~nm}$, with a polydispersity of between 0.4 and 0.5 , as measured using photon correlation spectrometry. Successive passes continued to reduce the average size, but the changes were quite small. Increasing the number of passes had no significant effect on the polydispersity.

Figure 2 shows the effect on the average liposome diameter of passing the phospholipid dispersions [10\% (wt/wt)] through the Microfluidizer a number of times at different operating pressures. The trends in the average diameter at the 3 operating pressures were approximately in parallel. Increasing either the operating pressure or the number of passes decreased the hydrodynamic diameter of the liposomes. It was evident that the effects of the pressure and the number of passes were additive. There was an approximately $40 \%$ decrease in the diameter between the largest (produced at 1 pass at $73 \mathrm{MPa}$ ) and the smallest (produced at 10 passes at $103 \mathrm{MPa}$ ) average liposome diameter measured.

The effect of phospholipid concentration on the average hydrodynamic diameter is shown in Figure 3. The dispersions containing 5 and $10 \%$ phospholipid showed a slightly larger reduction in average diameter after one pass through the Microfluidizer, but additional passes caused smaller size reductions in the $10 \%$ dispersion than in either the 1 or $5 \%$ phospholipid dispersions. This suggests that the $10 \%$ phospholipid dispersion was 


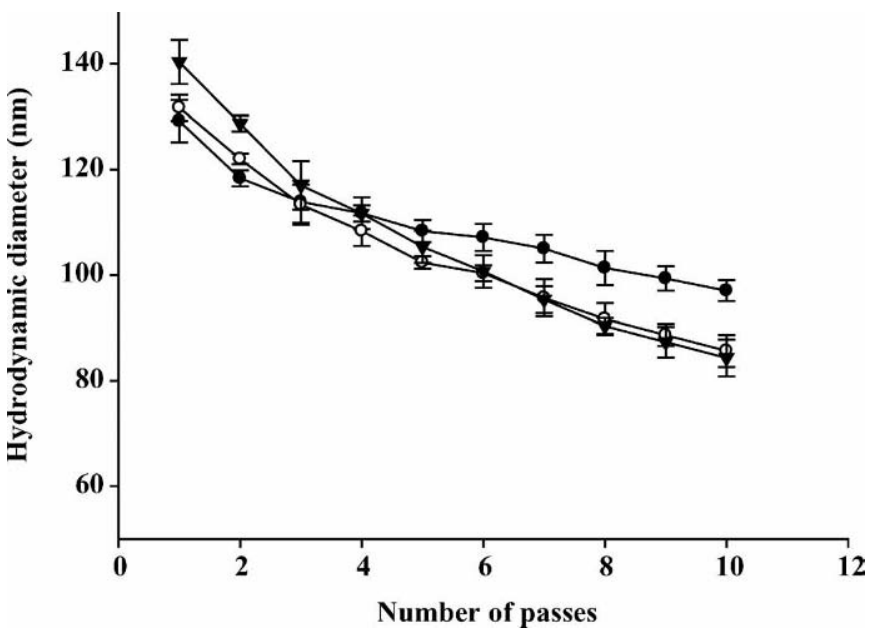

Figure 3. Effect of phospholipid concentration on average hydrodynamic diameter of liposomes formed during microfluidization at $103 \mathrm{MPa}$. The concentrations of the phospholipid dispersions were $10 \%(\bullet), 5 \%(\bigcirc)$, and $1 \%(\nabla)$. Each data point is the average of 3 or more replicates.

less sensitive to the shear and turbulence produced by the microfluidization process. This would be expected as the higher viscosity in more concentrated dispersions could resist deformation and breakup of liposome particles.

The size distribution for a $10 \%$ phospholipid liposome dispersion (5 passes through the Microfluidizer at 103 $\mathrm{MPa}$ ) was determined using asymmetrical flow fieldflow fractionation. The normalized response of the $90^{\circ}$ detector for various liposome diameters (as determined by light scattering) is shown in Figure 4. This provided

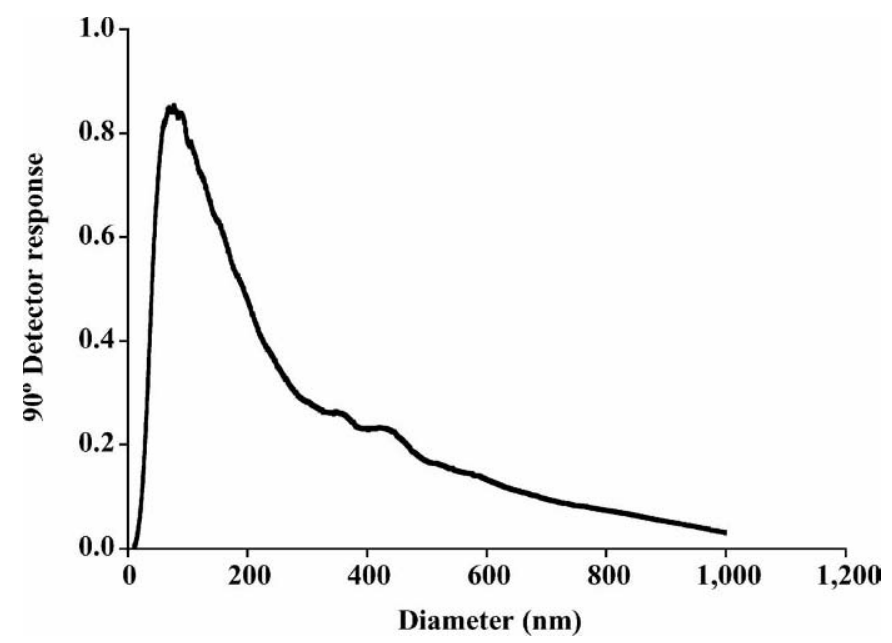

Figure 4. Normalized $90^{\circ}$ detector response for specified liposome diameters on a linear scale as determined by asymmetrical flow fieldflow fractionation. Data are from a 10\% phospholipid liposome dispersion passed through the Microfluidizer 5 times at a pressure of $103 \mathrm{MPa}$. an indication of the relative proportion of liposomes present for each diameter. However, care must be taken when interpreting the results as increasing the particle size results in increased scattering for the same number of particles.

The liposome dispersion had a primary peak between 50 and $100 \mathrm{~nm}$, with a long tail stretching past 1,000 $\mathrm{nm}$. The length of this tail is in agreement with the very high polydispersity values of 0.4 to 0.5 obtained using photon correlation spectrometry. However, because scattering intensity is proportional to diameter to the sixth power, it is unlikely that there were a significant number of the liposomes with an average diameter over $400 \mathrm{~nm}$.

The trends observed for the effects of pressure, number of passes, and phospholipid concentration on the average liposome size agree with those reported by Barnadas-Rodríguez and Sabés (2001). They used a smaller laboratory-scale Microfluidizer (model 110S) to investigate the effect of a number of production variables on liposome size. They found that the mean liposome diameter decreased with increasing pressure and number of passes. Bachmann et al. (1993) used a high-pressure homogenizer to produce liposomes and found that, although higher homogenization pressures and repeated recirculation led to reductions in vesicle diameter and heterogeneity, size reduction was less effective at phospholipid concentrations above $10 \%$. This supports the observations shown in Figure 3, with the 10\% phospholipid dispersion having a larger average diameter than lower phospholipid concentrations after 5 or more passes.

Koide and Karel (1987) were among the first to use a Microfluidizer (model M-110) to produce liposomes, reporting an average liposome diameter of $196 \mathrm{~nm}$ after 10 passes at $14 \mathrm{MPa}$ (140 bar). Peel (1999) used a highpressure homogenizer to process preformed multilamellar vesicles, and found that, after 5 passes at 13 $\mathrm{MPa}$ (130 bar), the average diameter stabilized at approximately $125 \mathrm{~nm}$. The diameters measured by these researchers were generally larger than those obtained in our experiments, presumably because of the lower pressures used. However, Brandl et al. (1998) used a high-pressure homogenizer at pressures of up to 140 MPa (20,000 psi or 1,400 bar), and obtained liposome dispersions with median diameters of $<40 \mathrm{~nm}$, which were much smaller than those found in our study.

The high polydispersity of liposome dispersions indicates a broad particle-size distribution, and is similar to the results of Zeisig and Cämmerer (2001), who reported that liposomes produced by microfluidization had an average diameter of 100 to $200 \mathrm{~nm}$ with a polydispersity of between 0.2 and 0.6 . 


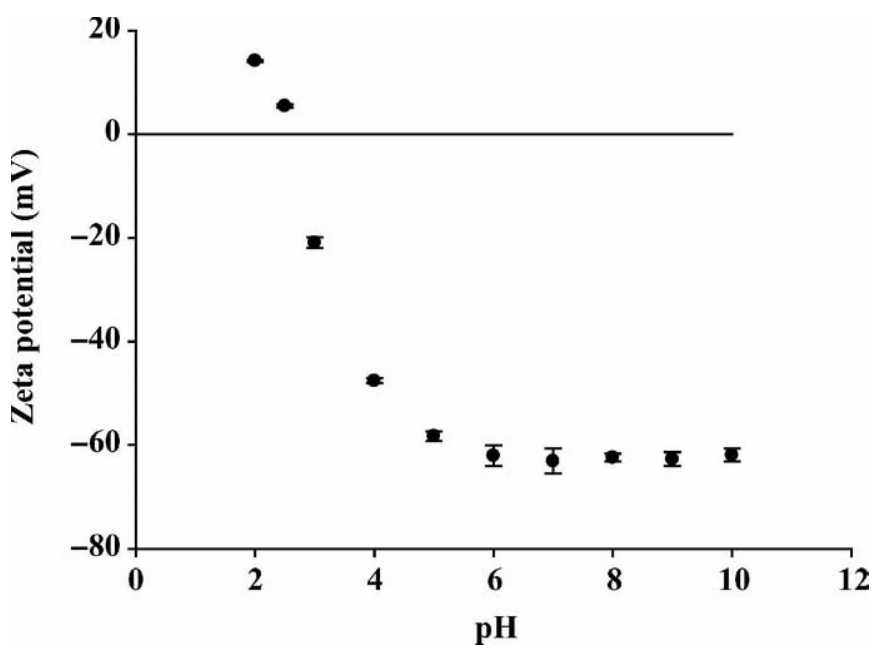

Figure 5. $\mathrm{pH}$ dependence of $\zeta$-potential of liposome dispersions. Each data point is the average of 3 or more replicates.

\section{$\zeta$-Potential}

The $\zeta$-potential values for liposome dispersions at various $\mathrm{pH}$ values are shown in Figure 5 . The liposome dispersions exhibited negative potentials at $\mathrm{pH}$ values above 3 , with a flat region at approximately $-65 \mathrm{mV}$ between $\mathrm{pH} 5$ and $\mathrm{pH} 10$. Below $\mathrm{pH} 5$, the $\zeta$-potential became rapidly less negative, with a potential of zero at $\mathrm{pH} 2.6$ and rising to a positive potential of $15 \mathrm{mV}$ at $\mathrm{pH}$ 2.0. Very few $\zeta$-potential values for liposomes have been reported in the literature, and the significant effect of the composition of the buffer salts used on the potential renders any comparison between different systems difficult. However, a $\zeta$-potential of $\sim-65 \mathrm{mV}$ is much larger than those of between 0 and $-43 \mathrm{mV}$ reported for other liposome dispersions (Talsma et al., 1989; Ruel-Gariepy et al., 2002). The negative $\zeta$-potential presumably arose because of the presence of negatively charged phospholipids, particularly PI, with the positive $\zeta$-potential below $\mathrm{pH} 3$ reflecting the positive charge of $\mathrm{PC}$ and $\mathrm{PE}$ molecules at these $\mathrm{pH}$ values.

Based on these results, it would be expected that liposomes produced from the MFGM phospholipid fraction would have a higher degree of charge repulsion than the liposomes commonly used in the literature, which may provide them with enhanced resistance to aggregation or coalescence.

\section{Microstructure}

In addition to simply confirming the presence of liposomes, electron microscopy can provide some valuable information regarding the size, shape, and structure of the liposomes within a dispersion. Negative-staining and thin-section transmission electron microscopy allows determination of liposome size at the very low end of the size spectrum, and can allow the lamellarity of a liposome dispersion to be estimated in a qualitative or semiquantitative manner (Perkins, 1993). Weiner (1995) stated that this was the method of choice below $5 \mu \mathrm{m}$. Cryo electron microscopy techniques are reported to be more likely to preserve the original structure of the liposomes (Weiner, 1995) and may provide threedimensional confirmation of the liposome structure.

Negative-Staining Transmission Electron Microscopy. The negative-staining technique was very quick and easy compared with the other 2 methods. A typical micrograph of the liposome dispersions is shown in Figure 6a. The liposomes could easily be identified as discrete particles that were predominantly spherical or rod-like in shape. The outer membrane surrounding the internal aqueous space could be clearly seen in many of the liposomes, and internal membranous structures could be identified. The micrographs contained a large number of very small particles $(\leq 40 \mathrm{~nm})$ interspersed with much larger particles (100 to $200 \mathrm{~nm}$ ).

Some of the liposome dispersions appeared to have irregular membranous structures trapped inside the outer bilayer (arrows in Figure 6b). Although multilamellar liposomes are usually thought of as being composed of neatly stacked lamellae at regular spacing, according to Perkins et al. (1993), most large heterogeneous liposome systems typically contain liposomes with irregularly spaced bilayers or "liposome within liposome" structures. These are perhaps more correctly referred to as multivesicular liposomes (Talsma et al., 1987; Brandl et al., 1998). Freeze-fracture transmission electron micrographs in Škalko et al. (1998) of liposomes produced via microfluidization also showed irregular-shaped vesicles trapped inside other vesicles, as did liposomes formed from phospholipid pastes produced by high-pressure homogenization (Brandl et al., 1998).

Thin-Section Transmission Electron Microscopy. A typical micrograph of the liposome dispersions produced using the standard thin section technique is shown in Figure 7a. Visual analysis of the images indicated that the liposomes were primarily between 80 and $100 \mathrm{~nm}$ in diameter. They appeared to be clumped together, with groups of densely packed liposomes surrounded by empty space. Many of the vesicles in the clumps seemed to be nonspherical, although the liposomes in the less-crowded areas had retained their spherical shape. This suggested that some of the nonspherical nature of the liposomes could be due to membrane deformation caused by the crowding within the liposome clumps. However, some liposomes appeared to be shrunken and collapsed, which did not seem to 
a

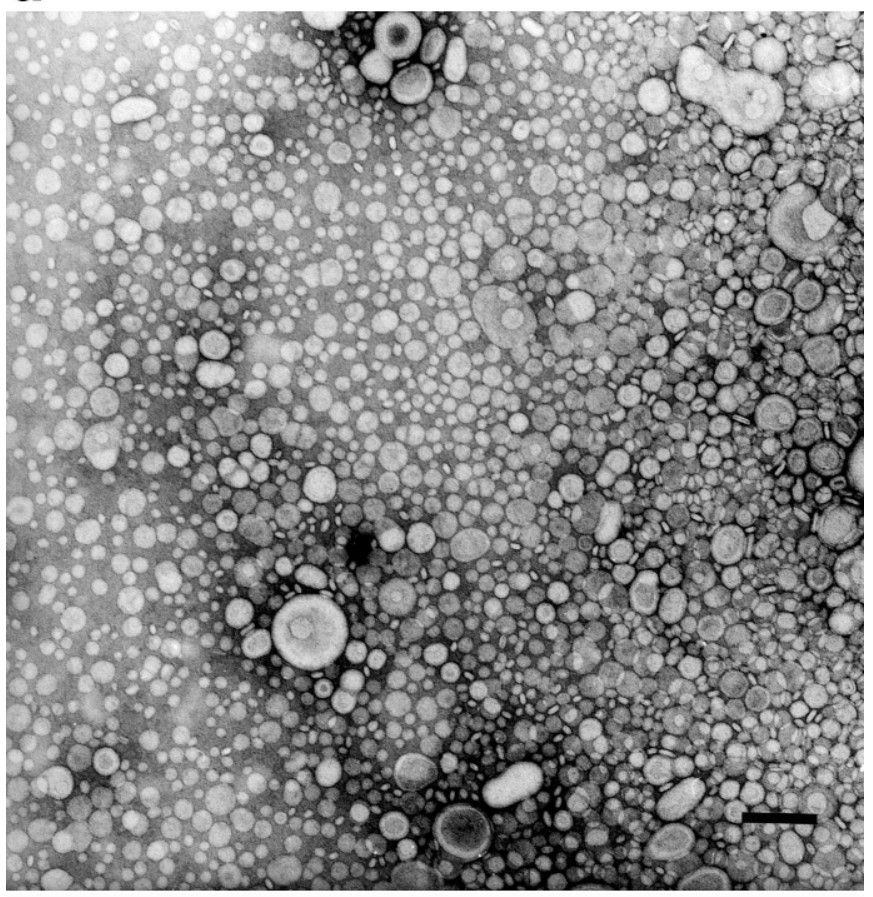

b

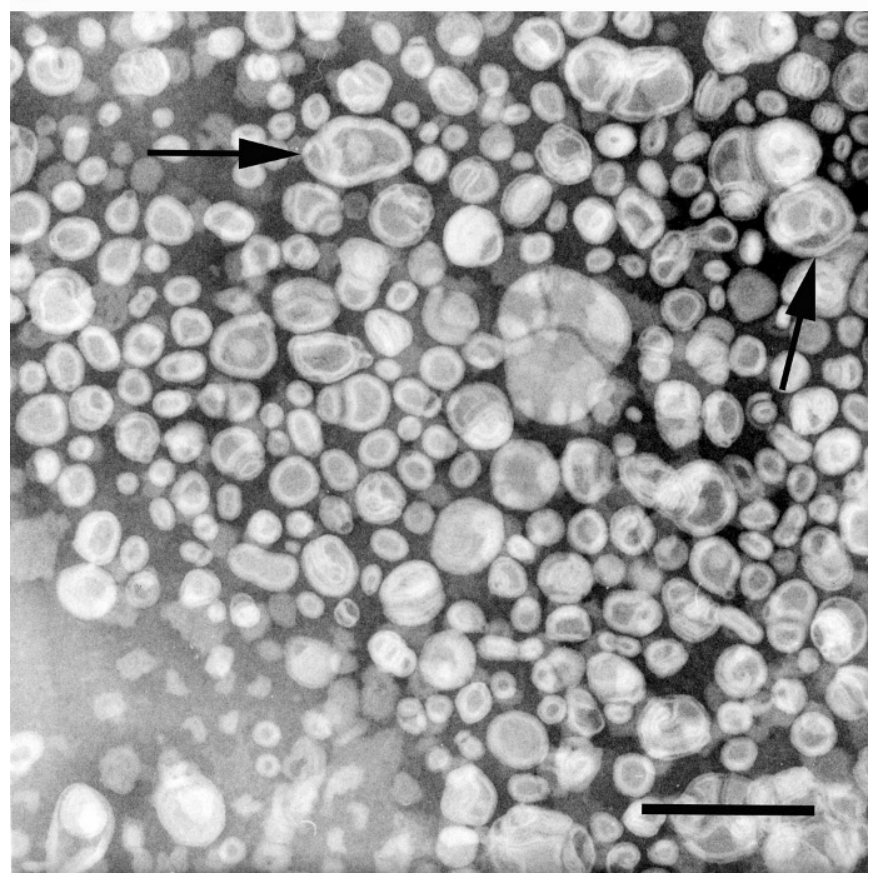

Figure 6. Two different magnifications (a and b) of negatively stained transmission electron micrographs of liposome dispersions. At the higher magnification (panel b) it is possible to see internal membranous structures as identified by arrows. Bar $=0.15 \mu \mathrm{m}$. a

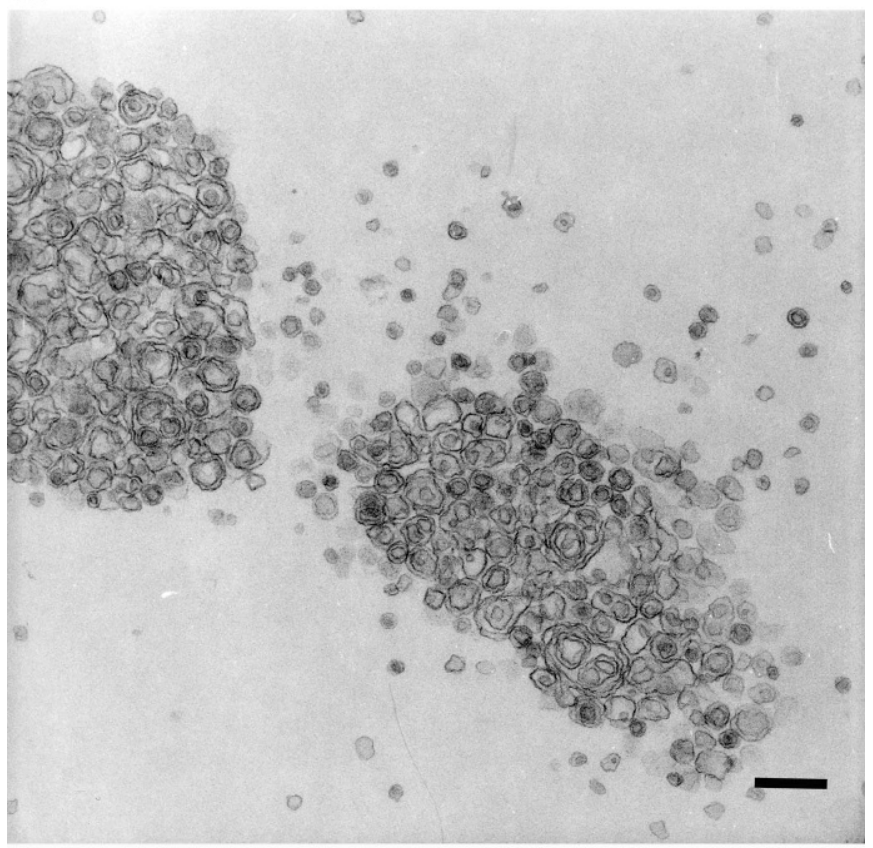

b

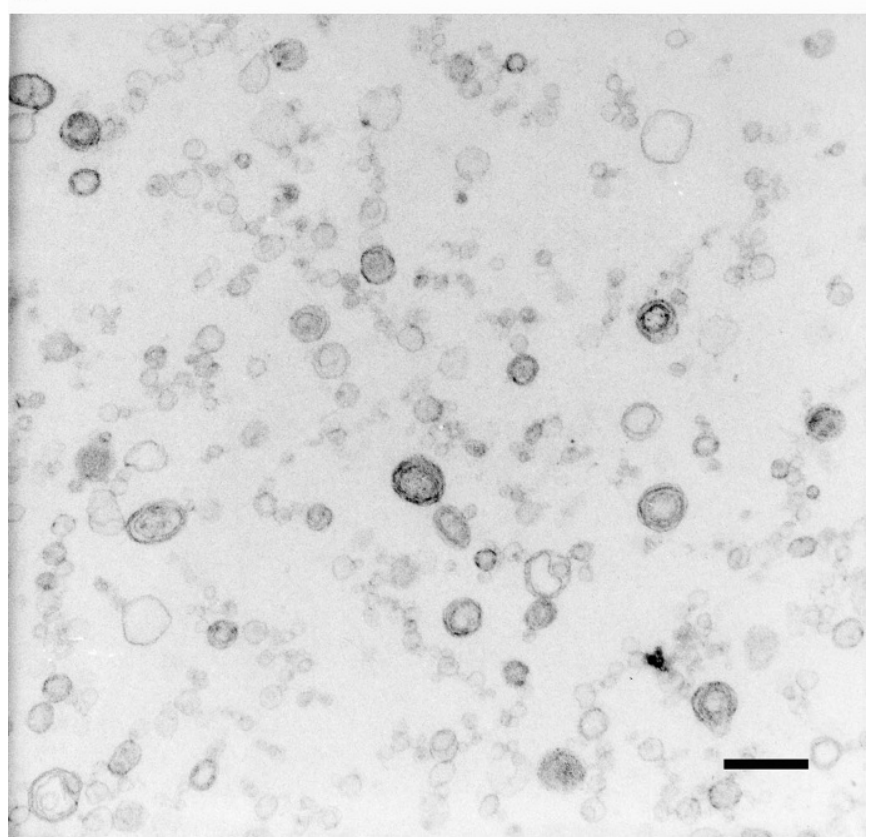

Figure 7. Thin-section transmission electron micrographs of liposome dispersions produced using a) acetone and b) ethanol during the dehydration step. Bar $=0.3 \mu \mathrm{m}$.

be because of tight packing. It seems likely that the dehydration steps used during preparation for this technique were responsible for the clumping of the liposomes, and may have contributed to the nonspherical nature of the vesicles. 


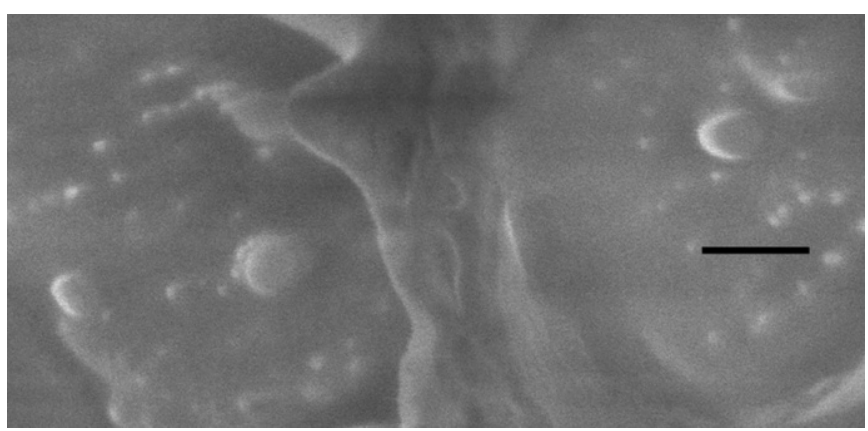

Figure 8. Cryo-field emission scanning electron micrograph of liposome dispersion. Bar $=0.3 \mu \mathrm{m}$.

An attempt was made to reduce the severity of the dehydration step by substituting acetone with ethanol at the same concentrations. A typical micrograph obtained using ethanol for the dehydration step is shown in Figure 7b. There was little or no clumping in any of the micrographs. However, many of the liposomes still appeared to be dehydrated and collapsed, although a number of intact spherical vesicles were present in each micrograph. The liposome diameters appeared to range from 50 to $150 \mathrm{~nm}$.

The apparent multilamellar nature of many of the liposomes was obvious, with others appearing to have only a single membrane. It was difficult to know whether these were indeed unilamellar liposomes, or whether the sample had been sectioned between the inner and outer membranes of those liposomes without cutting through the inner bilayers.

Cryo-Field Emission Scanning Electron Microscopy. The micrographs produced by this technique showed three-dimensional confirmation of smooth spheres consistent with that of liposomes (Figure 8). As expected, the approximately spherical vesicles had a wide particle size distribution, with both large and small liposomes evident in all dispersions. It was possible to see a number of large liposomes with diameters around $200 \mathrm{~nm}$ as well as some very small liposomes of approximately $40 \mathrm{~nm}$ in diameter.

\section{Effect of Processing on Phospholipid Oxidation}

There was no increase in either the peroxide value or the conjugated diene level in any of the phospholipid dispersions immediately after microfluidization or after storage for $1 \mathrm{wk}$ at $5^{\circ} \mathrm{C}$. This suggests that the processing conditions used to produce liposomes from the phospholipid material did not promote the oxidation reactions that lead to the formation of peroxides or conjugated dienes. This observation is in agreement with the results of Barnadas-Rodríguez and Sabés
(2001), who reported that microfluidization did not cause any increase in phospholipid oxidation.

Overall, this work showed that it is possible to produce liposomes from an MFGM phospholipid material using microfluidization. Electron microscopy revealed that a number of liposome structures were formed, including unilamellar, multilamellar, and multivesicular liposomes. The liposome dispersion had a broad particle size distribution, with an average hydrodynamic diameter of $\sim 95 \mathrm{~nm}$. Because of the origin of the phospholipids, the fatty acid profile of the MFGM phospholipids was more highly saturated than that of nonhydrogenated soy or egg phospholipid, which is likely to result in a higher phase transition temperature for the membrane and an increased resistance to oxidation. The high level of SM present in the phospholipid fraction may increase the stability of the liposome dispersion and reduce the membrane permeability. Initial experiments showed that the surface of these liposomes has a strong negative charge, which may further enhance their stability. Future studies will be undertaken to continue the characterization of the MFGM phospholipid liposomes, assessing permeability and phase transition temperature and comparing the results with liposome dispersions produced from soy phospholipids.

\section{ACKNOWLEDGMENTS}

This work was funded in part by the Foundation for Research, Science and Technology (FRST) and the Fonterra Co-operative Group Ltd. We thank the National School of Pharmacy at the University of Otago for providing the cryo-field emission scanning electron micrograph, Skelte Anema of Fonterra Innovation for his assistance with the particle size analysis, and Derek Haisman for helpful discussions.

\section{REFERENCES}

Ardhammer, M., P. Lincoln, and B. Norden. 2002. Invisible liposomes: Refractive index matching with sucrose enables flow dichroism assessment of peptide orientation in lipid vesicle membrane. Proc. Natl. Acad. Sci. USA 99:15313-15317.

Astaire, J. C., R. Ward, J. B. German, and R. Jimenez-Flores. 2003. Concentration of polar MFGM lipids from buttermilk by microfiltration and supercritical fluid extraction. J. Dairy Sci. 86:2297-2307.

Bachmann, D., M. Brandl, and G. Gregoriadis. 1993. Preparation of liposomes using a Mini-Lab $8.30 \mathrm{H}$ high-pressure homogenizer. Int. J. Pharmaceut. 91:69-74.

Barnadas-Rodriguez, R., and M. Sabes. 2001. Factors involved in the production of liposomes with a high-pressure homogenizer. Int. J. Pharmaceut. 213:175-186.

Betageri, G. V., and S. B. Kulkarni. 1999. Preparation of Liposomes. Pages 489-521 in Microspheres, Microcapsules and Liposomes. Vol. 1: Preparation and chemical applications. R. Arshady, ed. Citus Books, London, UK.

Blessing, T., J.-S. Remy, and J.-P. Behr. 1998. Monomolecular collapse of plasmid DNA into stable virus-like particles. Proc. Natl. Acad. Sci. USA 95:1427-1431. 
Boyd, L. C., N. C. Drye, and A. P. Hansen. 1999. Isolation and characterization of whey phospholipids. J. Dairy Sci. 82:2550-2557.

Brandl, M., M. Drechsler, D. Bachmann, C. Tardi, M. Schmidtgen, and K.-H. Bauer. 1998. Preparation and characterization of semisolid phospholipid dispersions and dilutions thereof. Int. J. Pharm. 170:187-199.

Chen, R. H., H. P. Win, and H. J. Fang. 2001. Vesicle size, size distribution, stability, and rheological properties of liposomes coated with water-soluble chitosans of different molecular weights and concentrations. J. Liposome Res. 11:211-228.

Corredig, M., and D. G. Dalgleish. 1998. Characterization of the interface of an oil-in-water emulsion stabilized by milk fat globule membrane material. J. Dairy Res. 65:465-477.

Corredig, M., R. R. Roesch, and D. G. Dalgleish. 2003. Production of a novel ingredient from buttermilk. J. Dairy Sci. 86:2744-2750.

Crook, T., W. Petrie, C. Wells, and D. Massari. 1992. Effects of phosphatidylserine in Alzheimer's disease. Psychopharmacol. Bull. 28:61-66.

Crook, T., J. Tinklenberg, J. Yesavage, W. Petrie, M. Nunzi, and D. Massari. 1991. Effects of phosphatidylserine in age-associated memory impairment. Neurology 41:644-649.

Diehl, B. 2001. High resolution NMR spectroscopy. Eur. J. Lipid Sci. Technol. 103:16-20.

Diehl, B. $2002 .{ }^{31} \mathrm{P}$ NMR in study of phosphorous-containing lipids. Lipid Technol. 14:62-65.

Fauquant, C., V. Briard, N. Leconte, and M. Michalski. 2005. Differently sized native milk fat globules separated by microfiltration: Fatty acid composition of the milk fat globule membrane and triglyceride core. Eur. J. Lipid Sci. Technol. 107:80-86.

Frezard, F. 1999. Liposomes: From biophysics to the design of peptide vaccines. Braz. J. Med. Biol. Res. 32:181-189.

Huwiler, A., T. Kolter, J. Pfeilschifter, and K. Sandhoff. 2000. Physiology and pathophysiology of sphingolipid metabolism and signaling. Biochim. Biophys. Acta 1485:63-99.

IUPAC. 1987. Standard Methods for the Analysis of Oils, Fats and Derivatives. 7th ed. IUPAC, Applied Chem Division, Commission on Oils and Fats Derivatives, Blackwell Scientific Publications, Oxford, UK.

Jackson, L. S., and K. Lee. 1991. Microencapsulation and the Food Industry. Lebensm. Wiss. Technol. 24:289-297.

Keenan, T. W., and I. H. Mather. 2002. Lipids-Milk Fat Globule Membrane. Pages 1568-1576 in Encyclopedia of Dairy Sciences. H. Roginski, J. W. Fuquay, and P. F. Fox, ed. Elsevier Science Ltd., Oxford, UK.

Kim, H. Y., and I. C. Baianu. 1991. Novel liposome microencapsulation techniques for food applications. Trends Food Sci. Technol. 2:55-61.

Koide, K., and M. Karel. 1987. Encapsulation and stimulated release of enzymes using lecithin vesicles. Int. J. Food Technol. 22:707-723.

Koopman, J., V. Turkish, and A. Monto. 1985. Infant formula and gastrointestinal illness. Am. J. Public Health 75:477-480.

Larivière, B., M. El Soda, Y. Soucy, G. Trépanier, P. Paquin, and J. Vuillemard. 1991. Microfluidized liposomes for the acceleration of cheese ripening. Int. Dairy J. 1:111-124.
Lasic, D. D. 1998. Novel applications of liposomes. Trends Biotechnol. 16:307-321.

Lethuaut, L., F. Métro, and C. Genot. 2002. Effect of droplet size on lipid oxidation rates of oil-in-water emulsions stabilized by protein. J. Am. Oil Chem. Soc. 79:425-430.

New, R. 1990a. Characterisation of liposomes. Pages 105-162 in Liposomes-A practical approach. R. New, ed. IRL Press, Oxford, UK.

New, R. 1990b. Preparation of liposomes. Pages 33-104 in Liposomes-A practical approach. R. New, ed. IRL Press, Oxford, UK.

Peel, M. 1999. Liposomes produced by combined homogenization/ extrusion. GIT Lab. J. 3:37-38.

Perkins, W. M., S. R. Minchey, P. L. Ahl, and A. S. Janoff, A.1993. The determination of liposome captured volume. Chem. Phys. Lipids 64:197-217.

Pheko, L., E. Chavez, and P. Lague. 1998. Effects of feeding flaxseed and probiotic supplementation to layers on egg composition and fatty acids. Department of Animal Science Research Reports, MacDonald Campus, McGill University, Montreal, Canada.

Picon, A., P. Gaya, M. Medina, and M. Nunez. 1994. The effect of liposome encapsulation of chymosin derived by fermentation on Manchego cheese ripening. J. Dairy Sci. 77:16-23.

Roesch, R. R., A. Rincon, and M. Corredig. 2004. Emulsifying properties of fractions prepared from commercial buttermilk by microfiltration. J. Dairy Sci. 87:4080-4087.

Ruel-Gariepy, E., G. Leclair, P. Hildgen, A. Gupa, and J.-C. Leroux. 2002. Thermosensitive chitosan-based hydrogel containing liposomes for the delivery of hydrophilic molecules. J. Control. Release 82:373-383.

Sato, H., H. Liu, I. Adachi, M. Ueno, M. Lemaire, and I. Horikoshi. 1994. Enhancement of the intestinal absorption of a cyclosporin derivative by milk fat globule membrane. Biol. Pharm. Bull. 17:1526-1528.

Škalko, N., J. Bouwstra, F. Spies, M. Stuart, P. Frederik, G. Gregoriadis. 1998. Morphological observations on liposomes bearing covalently bound protein: Studies with freeze-fracture and cryo electron microscopy and small angle X-ray scattering techniques. Biochim. Biophys. Acta 1370:151-160.

Talsma, H., H. Jousma, K. Nicolay, and D. Lasic. 1987. Multilamellar or multivesicular vesicles? Int. J. Pharm. 37:171-173.

Talsma, H., A. Özer, L. van Bloois, and D. Crommelin. 1989. The size reduction of liposomes with a high-pressure homogenizer (Microfluidizer). Characterization of prepared dispersions and comparison with conventional methods. Drug Dev. Ind. Pharm. 15:197-207.

Watwe, R., and J. Bellare. 1995. Manufacture of liposomes: A review. Curr. Sci. 68:715-724.

Weiner, N. 1995. Phospholipid liposomes: Properties and potential use in flavor encapsulation. Pages 210-218 in Flavor Technology. Vol. 610. American Chemical Society Symposium Series. American Chemical Society, Washington, DC.

Yuasa, H., M. Sekiya, S. Ozeki, and J. Watanabe. 1994. Evaluation of milk fat-globule membrane (MFGM) emulsion for oral administration: Absorption of alpha-linolenic acid in rats and the effect of emulsion droplet size. Biol. Pharm. Bull. 17:756-758.

Zeisig, R., and B. Cämmerer. 2001. Liposomes in the food industry. Pages 101-119 in Microencapsulation of Food Ingredients. P. Vilstrup, ed. Leatherhead Publishing, London, UK. 